\title{
Performance comparison of proposed scheme coded DWT-OFDM system with that of Conventional OFDM
}

\author{
SIMMI GARG $^{1 *}$, ANUJ KUMAR SHARMA $^{2}$, ANAND KUMAR TYAGI $^{3}$ \\ ${ }^{1}$ I K Gujral Punjab Technical University, Kapurthala, Punjab, INDIA \\ ${ }^{2}$ LRDAV College, Jagraon, Punjab, INDIA \\ ${ }^{3}$ Shaheed Bhagat Singh State Technical Campus, Ferozepur, Punjab, INDIA
}

\begin{abstract}
In this paper, we have investigated the Proposed scheme coded DWT-OFDM system in comparison to conventional OFDM system over AWGN channel. 16, 32, 64 - QAM and PSK modulation schemes have been considered to evaluate the system. Moreover, two different wavelets have been used for examining the system. Simulations are performed using MATLAB software. It is found that proposed scheme based DWT-OFDM system performs better than conventional OFDM system under all the considered modulation schemes.
\end{abstract}

Keywords:- OFDM, MCM,DWT, FFT, QAM, PSK

Received: April 25, 2019. Revised: May 7, 2020. Re-Revised: May 31, 2020. Accepted: June 20, 2020. Published: July 10, 2020.

\section{Introduction}

An alarming escalation of demand for high data rate multimedia based services and high spectral efficiency are the major necessities for the continued technology development in future wireless communications. It is obvious that the existing $3 \mathrm{G}$ wireless systems will be incapable to carry with this ever increasing demand for wireless services. The next generation wireless communication systems fourth generation (4G), LTE, and fifth generation $(5 \mathrm{G})$ are expected to support much higher data rate services. Several advancements have been integrated for improvement of the data rate and the system performance.

In recent years, a significant development of telecommunications can be observed. In particular, wireless solutions have become very popular, because they enable their users to communicate in a wide variety of places. In order to satisfy all the needs of modern consumers, wireless communication has to face nowadays several challenges. Orthogonal Frequency Division multiplexing (OFDM) is a multi-carrier modulation (MCM) technique which has been found to be very effective for communication systems. OFDM scheme divide the available channel bandwidth into low bandwidth sub-channels.

In the OFDM technique, a high-bit-rate data stream is split into $\mathrm{N}$ parallel low-bit rate data channel [1]. The parallel data streams are transmitted simultaneously on different carrier frequencies. This enables the channel to be robust against multi-path propagation [2]. OFDM scheme consist of high data capacity and mitigation to interference patterns. Moreover, subchannels are orthogonal to each other. But, the Loss of orthogonality between the sub-channels leads to deterioration of the signal. There are many advantages of implementing OFDM systems. The difference between the frequencies of adjacent subcarriers in OFDM systems is chosen in such a way that the subcarriers are orthogonal over the symbol duration. Therefore, the data transmitted on parallel subcarriers can be recovered despite the fact that the spectra of the transmitted signals overlap. OFDM systems are more robust to Inter-channel interference, inter-carrier interference. Narrow band width effects are very low. OFDM is more resistant to frequency selective fading than single carrier systems. OFDM is very resilient to inter-symbol and inter-frame interference. This is due to the fact that each of the sub-channel carries low data rate data stream. Interference appearing on a channel may be bandwidth limited and in this way it does not affect all the sub-channels. This reduces the channel fluctuation.

OFDM suffers from many disadvantages. Sensitivity to carrier frequency offset, High peak to average power ratio, receiver complexity are the main disadvantages of OFDM systems. OFDM signals are characterized by noise like amplitude variation in time domain and have relatively large dynamic range leading to high peak to average power ratio (PAPR). 
Complexity of the OFDM receiver increases with higher number of sub-channels. The available frequency band is used in a very effective way due to the fact that the sub-channels spectra overlap and the OFDM signal has a noise-like spectrum. A comparison between DCT and DFT based OFDM systems been studied [3].

In order to improve the system performance, signals are studied in time and frequency domain simultaneously using Wavelet transforms [4, 5]. Wavelets have many advantages over conventional FFT-OFDM systems. Wavelet based systems are more robust against carrier frequency offset, inter symbol interference and inter channel interference [6]. Hence, DWT based systems leads to reduction in BER as compare to conventional OFDM systems. Comparison between conventional and wavelet based OFDM has been studied [7]. Moreover, the impact of AWGN channel on the performance of conventional and wavelet based OFDM has also been considered [8]. System performance can be improved by using error correction codes [9]. Error correction codes involve sending the redundant bits along with the information bits. These redundant bits helps to combat the errors introduced during transmission of the signal from one end to another [10]. The performance of FEC based OFDM system using MPSK modulation has been discussed in [11]. Till date, various error correction codes are developed namely Convolution codes, Low density parity check codes, Turbo codes, Golay codes etc. Performance of wavelet based system using different coding schemes has been studied and relative comparison has been considered [12].

Convolution codes consist of shift registers and modulo2 adder [13]. Here, the output of the convolution encoder depends on the incoming bit as well as on the previous bits. The contribution of the previous bits depends on the generator polynomial used for simulating the system [14]. The received data is decoded at the receiver side to reconstruct the original signal. BER performance of convolution coded OFDM system has been considered and results depicted that Convolution coded system performs better than conventional OFDM systems [15]. [16] Studied the performance of DFT-OFDM, DCT-OFDM and DWTOFDM systems in AWGN channel and found that DWT-OFDM system outperforms the other systems.

Researchers are continuously working to enhance the performance of the convolution code based systems. Simmi Garg et al. have proposed an error correction code that can lead to improvement in the performance of the communication system [17]. Authors have investigated the relative performance of the Proposed scheme coded DWT-OFDM with Convolution scheme coded DWT- OFDM system and results depicted that proposed scheme based DWT-OFDM system outperform the Convolution scheme coded DWTOFDM system. In this present paper, authors have studied the performance comparison of proposed scheme coded DWT-OFDM with that of conventional OFDM systems. System model has been explained in section 2. Section 3 explains the results and discussions while section 4 concludes the paper.

\section{System Model}

Proposed scheme is implemented on the input data stream. Coded data is then modulated using modulation scheme under consideration. Inverse discrete wavelet transform is done of the modulated data. This converts the frequency frame to time frame. After this, the data is send on the communication channel. Due to various channel impairments, the data gets corrupted and this corrupted data is received by the receiver. At the receiver side, the first discrete wavelet transform (DWT) is performed on the received data that convert time domain signal into frequency domain signal. After, this demodulator performs its function and demodulates the data. The demodulated data is sent to the decoder in order to retrieve original information. Conventional OFDM system has been simulated by replacing IDWT and DWT block with IFFT and FFT blocks.

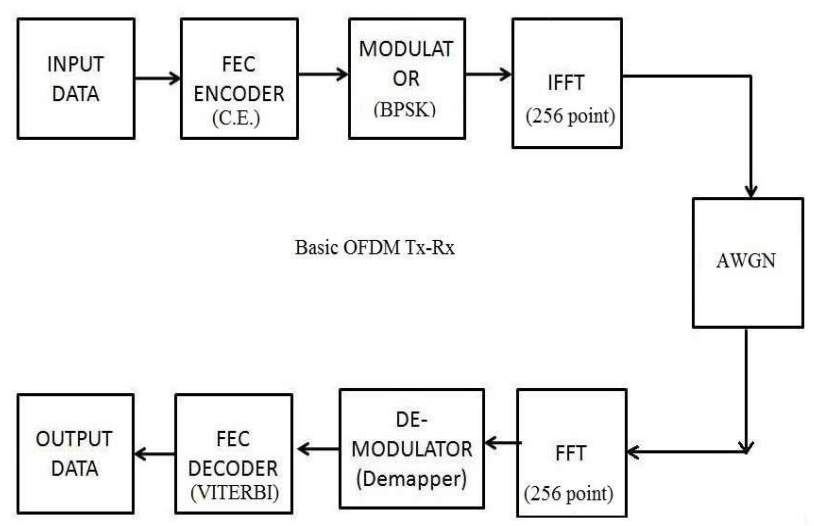

Fig. 1 Block model of encoded FFT-OFDM

\section{Results and Discussions}

Performance of the system is evaluated using MATLAB simulations. Input data stream is encoded using proposed scheme. Then, encoded data is modulated. We have used two types of modulation schemes namely QAM and PSK (and their higher orders). After this, IDWT of modulated data is performed using Haar and Symlet 5 wavelets. AWGN channel is used for transmission of data stream. After the insertion of noise by the transmission channel, DWT of received data is done. After this received data is demodulated. Demodulated data is decoded. For simulating conventional OFDM, IFFT and FFT operations are performed. Bit Error rate (BER) performance of the system is studied as the function of $\mathrm{SNR}(\mathrm{Eb} / \mathrm{No}$ in $\mathrm{dB})$ over AWGN channel. For FFTOFDM system, IDWT and DWT blocks are replaced by IFFT and FFT blocks respectively. Cyclic prefix of 
length one fourth of number of data sub carriers are used.

Table 1. Parameters used

\begin{tabular}{|l|l|l|}
\hline Parameter & $\begin{array}{l}\text { Proposed scheme } \\
\text { coded DW- } \\
\text { OFDM }\end{array}$ & FFT-OFDM \\
\hline $\begin{array}{l}\text { Number of } \\
\text { input bits }\end{array}$ & 128000 & 128000 \\
\hline Wavelet used & Haar, Symmlet5 & - \\
\hline $\begin{array}{l}\text { Type of } \\
\text { modulation }\end{array}$ & $\begin{array}{l}16,32,64-Q A M \\
\text { and 16,32, 64- } \\
\text { PSK }\end{array}$ & $\begin{array}{l}16,32,64-Q A M \\
\text { and 16,32, 64- } \\
\text { PSK }\end{array}$ \\
\hline Channel & AWGN & AWGN \\
\hline Code Rate & $1 / 2$ & - \\
\hline Eb/No(in dB) & $1-20$ & $1-20$ \\
\hline
\end{tabular}

\subsection{Comparative performance of proposed} system using 'Haar' wavelet over AWGN channel Figure 2 represents the performance comparison of proposed scheme coded DWT-OFDM with FFT-OFDM using 16-QAM modulation under AWGN channel. Results depicts that Proposed system outperform the conventional FFT-OFDM. For example, in order to achieve BER of $10^{-3}$, proposed system requires $10 \mathrm{~dB}$ of Eb/No while conventional FFT-OFDM system requires approximately $16 \mathrm{~dB}$ value.

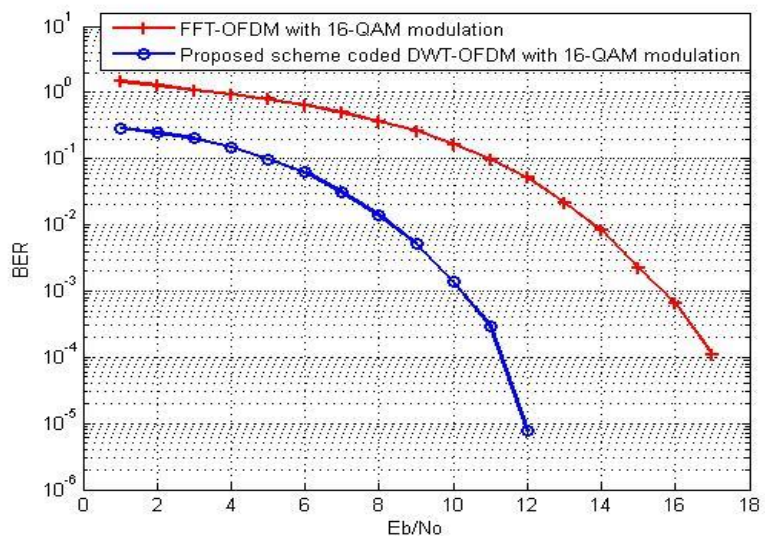

Fig. 2 Performance comparison of Proposed DWTOFDM with FFT-OFDM using 16-QAM modulation.

Figure 3 and 4 depicts the relative performance of proposed scheme coded DWT-OFDM system and conventional FFT-OFDM using 32-QAM and 64 QAM modulation respectively. AWGN channel has been considered for simulations. This is clear from the results obtained that performance of the proposed system is better than conventional FFT-OFDM system for both types of modulation schemes.

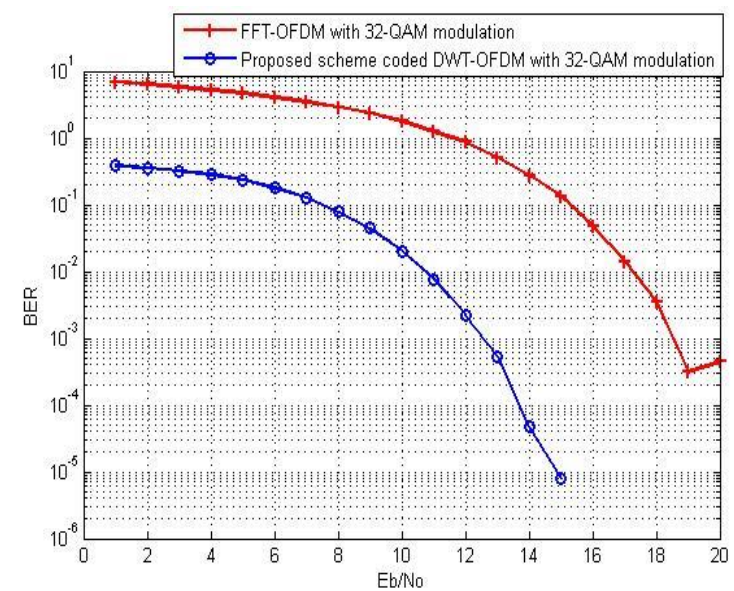

Fig. 3Performance comparison of Proposed DWTOFDM with FFT-OFDM using 32-QAM modulation

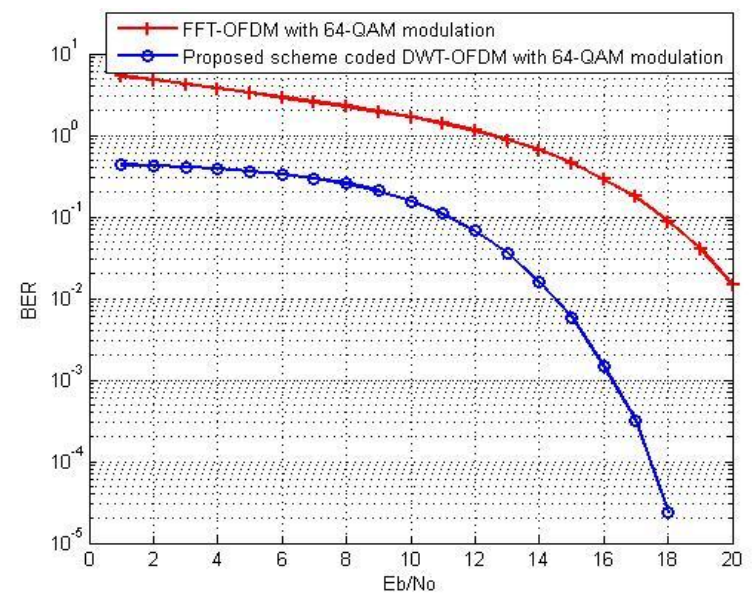

Fig. 4 Performance comparison of Proposed DWTOFDM with FFT-OFDM using 64-QAM modulation

Moreover, the Proposed system has been studied using different PSK modulations over AWGN channel. Figure 5 shows that performance of Proposed scheme coded DWT-OFDM with FFTOFDM system using 16-PSK modulation over AWGN channel. Haar wavelet has been considered for simulations. Proposed system outperforms the conventional FFT-OFDM over entire range of $\mathrm{Eb} /$ No considered. For example, in order to achieve BER of $10^{-3}$, Proposed system requires $13 \mathrm{~dB}$ of $\mathrm{Eb} / \mathrm{No}$ value while conventional FFT-OFDM system needs $19 \mathrm{~dB}$ of Eb/No value. 


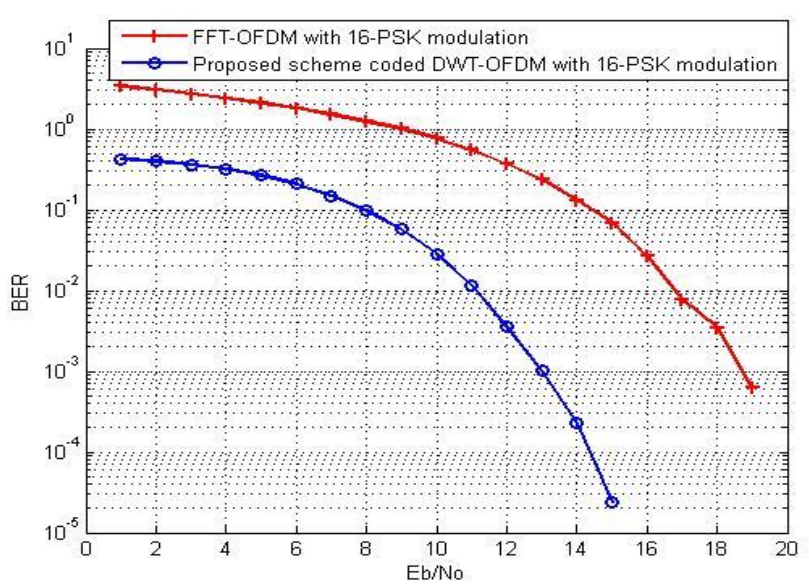

Fig. 5 Performance comparison of Proposed DWTOFDM with FFT-OFDM using 16-PSK modulation

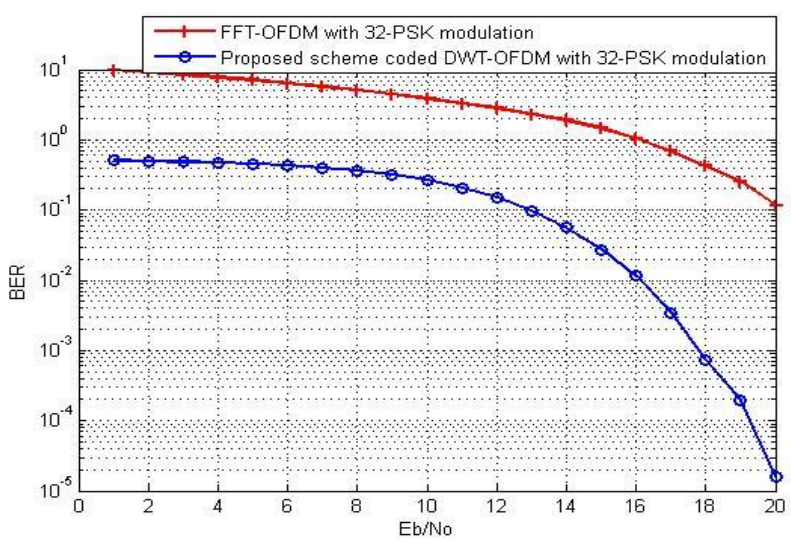

Fig. 6 Performance comparison of Proposed DWTOFDM with FFT-OFDM using 32-PSK modulation

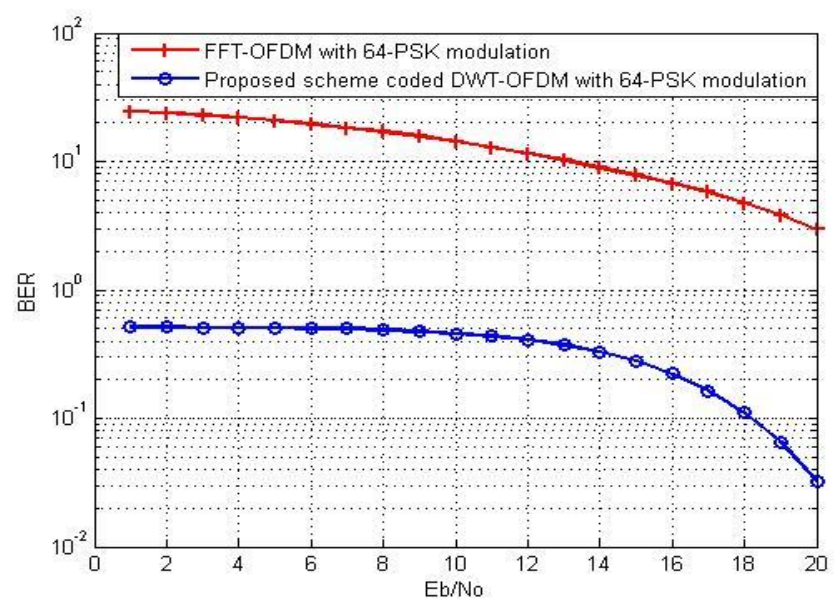

Fig. 7 Performance comparison of Proposed DWTOFDM with FFT-OFDM using 64PSK modulation.

Figure 6 and 7 shows the performance comparison of proposed system with that of conventional FFT-OFDM system using 32 and 64 PSK modulation scheme over AWGN channel. Here again, Proposed scheme coded DWT-OFDM system found to outperform the conventional system.

\subsection{Comparative performance of proposed} system using Symmlet 5 wavelet over AWGN channel
Figure 8 represents the performance comparison of Proposed scheme coded DWT-OFDM system using Symmlet 5 wavelet over AWGN channel with that of conventional FFT-OFDM system. The modulation scheme used for the simulations is 16 QAM. It is clear from the figure 8 that Proposed system outperform the conventional FFT-OFDM system over the entire range of $\mathrm{Eb} /$ No. For example, proposed system achieves BER of $10^{-2}$ at $\mathrm{Eb} / \mathrm{No}$ value of $8 \mathrm{~dB}$ while conventional FFT-OFDM achieves the same at $14 \mathrm{~Eb} / \mathrm{No}$ value. Hence, it can be concluded that proposed system leads to reduction in BER value with Symmlet 5 wavelet and 16 QAM modulation.

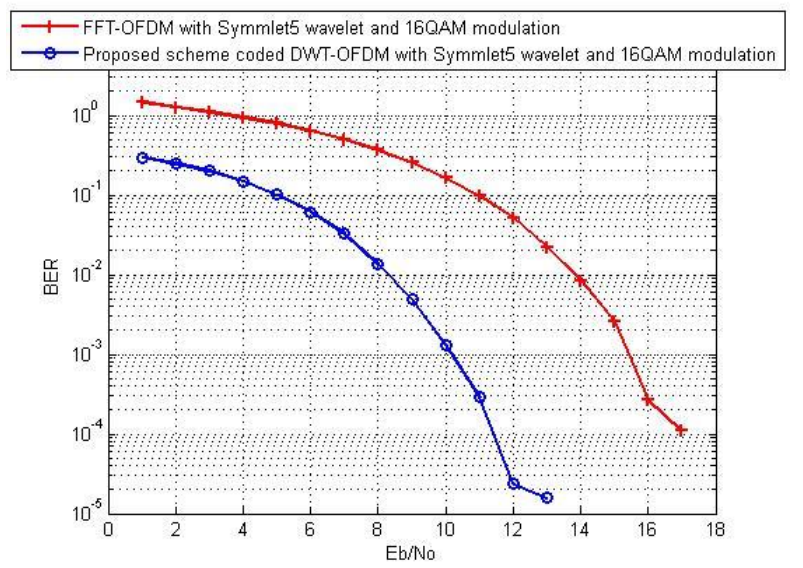

Fig. 8 Performance comparison of Proposed DWTOFDM with FFT-OFDM using 16-QAM modulation.

Figure 9 and 10 represents the Proposed scheme coded DWT-OFDM with FFT-OFDM system using 32 QAM and 64 QAM modulation with Symmlet 5 wavelet over AWGN channel. It is clear from the results that proposed system performs better than conventional system with both modulation schemes with symmlet wavelet.

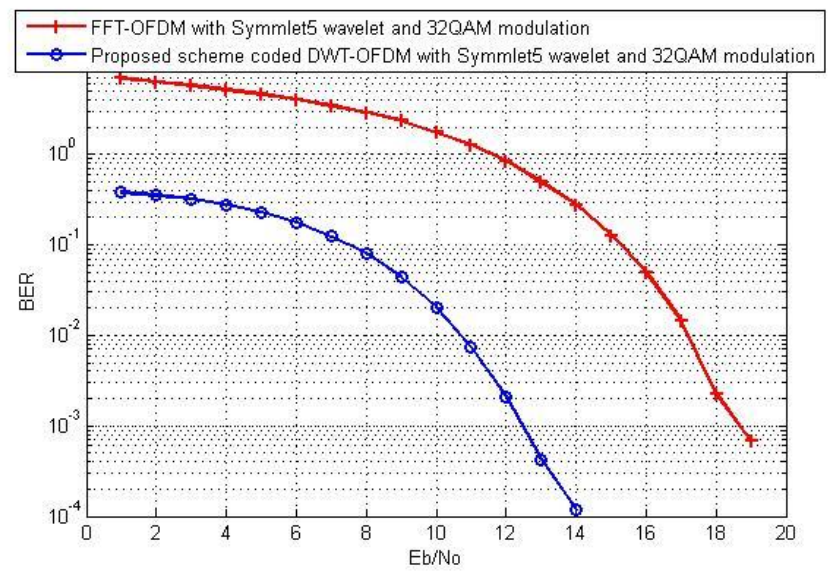

Fig. 9 Performance comparison of Proposed DWTOFDM with FFT-OFDM using 32-QAM modulation. 


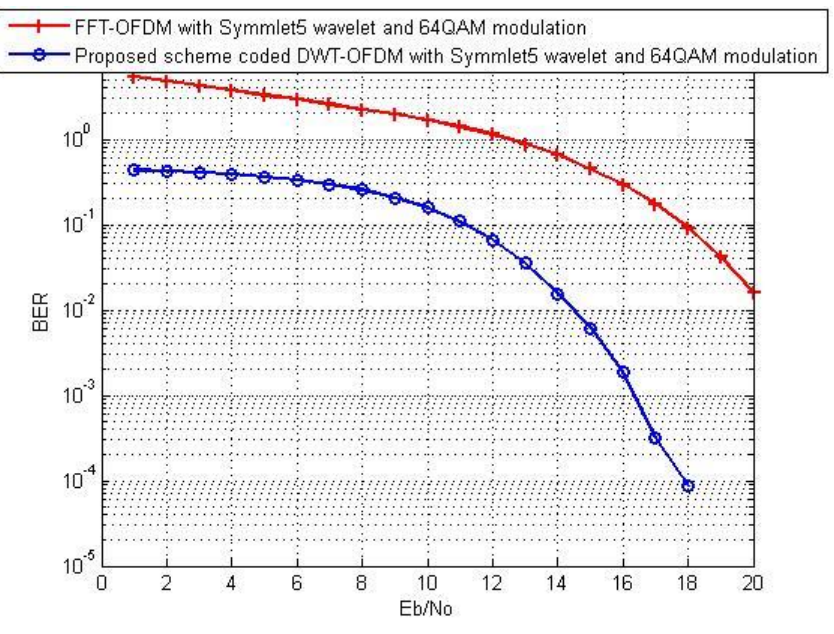

Fig. 10 Performance comparison of Proposed DWTOFDM with FFT-OFDM using 64-QAM modulation. Moreover, the system has been studied using 16, 32 and 64 PSK modulation with symmlet 5 wavelet over AWGN channel. Figure 11-13 denotes the results obtained after the simulations using 16, 32 and 64 PSK modulation. Here again, proposed system outperforms the conventional system with all the three type of PSK modulations.

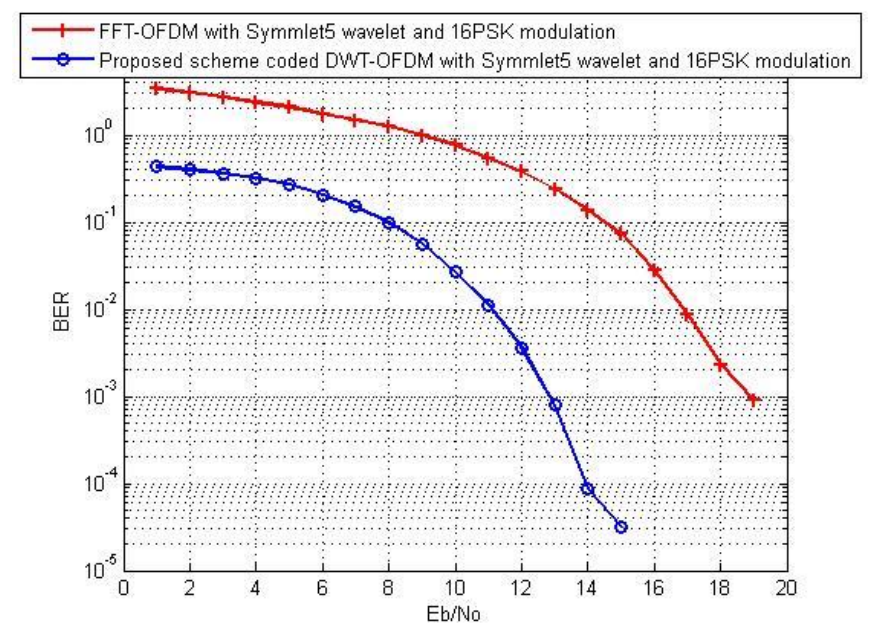

Fig. 11 Performance comparison of Proposed DWTOFDM with FFT-OFDM using 16-PSK modulation.

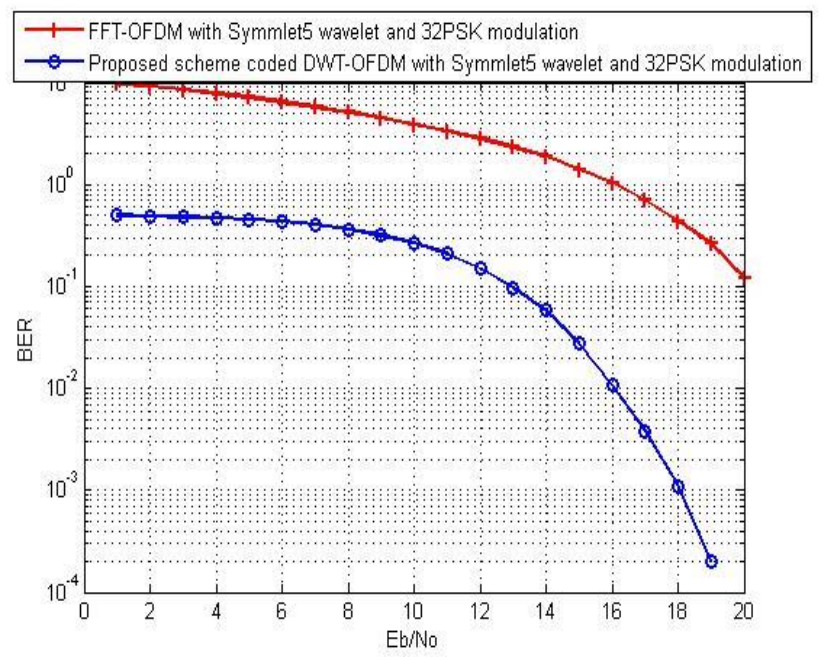

Fig. 12 Performance comparison of Proposed DWTOFDM with FFT-OFDM using 32-PSK modulation.

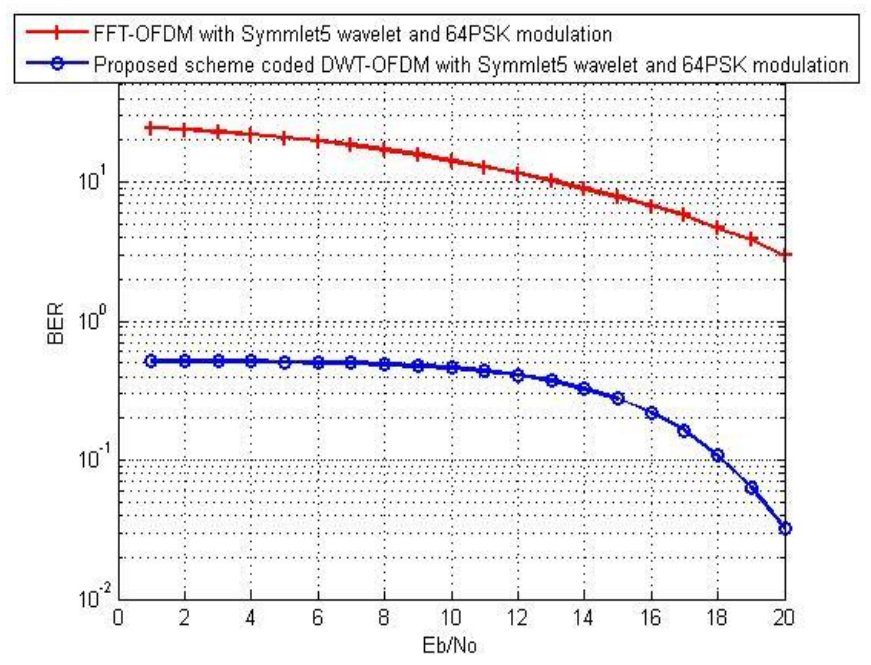

Fig. 13 Performance comparison of Proposed DWTOFDM with FFT-OFDM using 64 PSK modulation.

From the results obtained from figures 11-13, this can be concluded that proposed scheme coded DWTOFDM system performance is much better than conventional system. Proposed system leads to reduction in BER to a large extent.

\section{Conclusion}

In this paper, earlier proposed error correction scheme is compared with conventional OFDM system. Performance of the system has been evaluated using 16, 32, 64 QAM and PSK modulation schemes. Results depict that Proposed scheme coded DWT-OFDM system outperforms the conventional OFDM with all types of modulation schemes considered. Moreover, performance of the proposed system, with both Haar and Symmlet 5 wavelet, found to be better than conventional OFDM system.

\section{Acknowledgements}

Authors are thankful to anonymous reviewers for their careful reading and useful comments for the improvement of the present reserch work. Also, authors gratefully acknowledge the support provided by the Punjab Technical University, Kapurthala, Punjab, India

\section{References}

[1] Engels M, Wireless OFDM Systems: How To Make Them Work?, Kluwer Academic Publishers, 2002

[2] Satzberg B R., "Performance of an efficient parallel data transmission system," IEEE Transactions on Communication Technology, vol. 15 , no. 6, pp. 805-811, 1967

[3] P. Tan and N. C. Beaulieu, "A comparison of DCT-based OFDM and DFT-based OFDM in frequency offset and fading channels," IEEE Transactions on Communications, vol. 54,no. 11,pp. 2113-2125, 2006

[4] B.G. Negash and H. Nikookar, "Wavelet based Multicarrier Transmission over Multipath 
Wireless Channels", Electronic letters, vol. 36, pp.1787-1788, 2000

[5] M. Lakshmanan and H. Nikookar, "A Review of Wavelets for Digital Wireless Communication", Wireless Personal Communications, vol.37, pp. 387-420, 2006

[6] L. Debnath and J.P. Antoine, "Wavelet Transforms and Their Applications", Physics Today, Vol. 56, No. 4, pp.68, 2003

[7] M.K. Gupta and S. Tiwari, "Performance evaluation of Conventional and Wavelet based OFDM system", International Journal of Electronics and Communications, vol. 67, pp. 348-354, 2013

[8] V. Sharma and G. Singh, "BER assessment of conventional OFDM and wavelet based OFDM over AWGN channel", Optic, vol.125, pp. 60716073, 2014

[9] F.J. MacWilliams and N.J.A. Sloane, The theory of error correcting codes, North Holland Publishing Company, Amsterdam, 1977

[10] Bossert M., Channel Coding for Telecommunications, John Wiley and Sons, New York, 1999

[11] Kansal L., Sharma V., and Singh J., "BER assessment of FEC incorporated OFDM-MPSK wireless system", Proceedings of Fifth International conference on advance computing and communication technologies, India, 21-22 feb,2015

[12] Zhang H., Yuan D., Jiang M., Wu D., "Performance comparison of W-OFDM with different coding schemes" Procedings IEEE radio and wireless conference RAWCON, Boston, 2003, pp. 59-62

[13] A. Viterbi, "Convolutional codes and their performance in communication systems", IEEE Transactions on Communication Technology, vol. 19, pp. 751-772, 1971

[14] J. Rosenthal, J.M. Schumacher and E.V. York, "On behaviors of convolutional codes", IEEE Transactions on Information Theory, vol. 42, no. 6, pp.1881, 1996

[15] Hader N., Bhuyan M., Ahmed M., Faisel M., and Salauddin M., "Bit Error rate performance analysis of Convolutional coded orthogonal frequency division multiplexing system", Proceedings of $57^{\text {th }}$ international scientific conference on power and electrical engineering of Riga technical university, 13-14 october, 2016

[16] D Gupta, B.V. Vats V B, and Garg K K, "Performance analysis of DFT-OFDM, DCTOFDM, and DWT-OFDM systems in AWGN channel," in Proceedings of the 4th International Conference on Wireless and Mobile Communications (ICWMC '08), pp. 214-216, Athens, Greece, August 2008
[17] S. Garg, A.K. Sharma, A.K. Tyagi, "BER reduction in DWT-OFDM systems using a Novel Error Correction Scheme", Research \& Reviews: Journal of Physics, Vol. 8, No. 2, 2019

\section{Creative Commons Attribution License 4.0 (Attribution 4.0 International, CC BY 4.0)}

This article is published under the terms of the Creative Commons Attribution License 4.0 https://creativecommons.org/licenses/by/4.0/deed.en $\underline{\mathrm{US}}$ 